\title{
Research on Functional Electrical Stimulation to Achieve Hand Gripping Action
}

\author{
Xin-qi HE, Li-juan SHI, Liu-yang XU, Yi YU, \\ Yun ZHAO and Jun-Tang LIN
}

Xinxiang medical university, Henan, China

Keywords: Cerebral stroke, Hand gripping action, Functional electrical stimulation

\begin{abstract}
Functional Electrical Stimulation is a treatment method of using the output current of the functional electric stimulator to stimulate the nerves or muscles through the setting of functional electrical stimulator parameters. The motor function of the injured patients recovery the function of movement of a treatment method, which is for the treatment of the most common approach to patients caused by motor dysfunction due to damage to the nerves or muscles. There is no treatment method in medicine since the cerebral stroke caused by limb motor dysfunction, for those with normal thinking but the neural pathways blocked, the functional electrical stimulation is a promising technology in rehabilitation engineering, which can successfully recover partial motor function in patients with partial denervation. This experiment through the A-M functional electrical stimulator to adjust the output current amplitude, according to the capacity of the hand and forearm muscles giving appropriate electrical stimulation, and ultimately achieve the bending and stretching of the single finger, curving of two fingers and three fingers.
\end{abstract}

\section{Introduction}

Cerebral apoplexy, as known as stroke in our daily life, a common cause for the head or broken blood vessels caused by external causes of cerebral vascular congestion, because of the blood can't flow smoothly into the head leading to brain tissue damaged. Due to transfer information between nerves and muscles in the brain was damaged, the process of the effective implementation of cerebral apoplexy patients and exercise capacity is reduced, resulting in the out of the control. According to the incomplete statistics, Currently, incidence of stroke in $185 \sim 219 / 10$. With the gradually entered the aging society in our country, our country will increase the number of the patients with cerebral apoplexy. At the same time, the survival rate of the patients with cerebral apoplexy is about three forths, and all of them cannot provide daily life for themselves. At the same time, they also can have different degrees of movement disorders or with advanced brain dysfunction such as all kinds of complications. In recent decades, as a pursuit of the healthy life, how to solve in patients with cerebral apoplexy sequela, improving the quality of the life of cerebral apoplexy patients attract people to pay more attention to it. Now, it has become one of the research topics in recent decades. For many patients with damaged neural pathways, functional electrical stimulation technology has been gradually mature and begin to used in the treatment of stroke patients.

\section{Materials and Methods}

Functional electrical stimulation is a good choice. According to output current to stimulate nerve or muscle, making to regain their motor function in patients with damage to motor function a treatment. This experiment adopts A - M Systems 
Isolated Pulse Stimulator 2100 electrical Stimulator, as shown in figure 1, by setting the delay, Pulse duration, Pulse interval, Pulse width and the current amplitude to stimulate muscles to realize the hand grasping movements. The control part of the Isolated Pulse Stimulator of 2100 is divided into three modules, the beginning, timing control and output module. Start module includes trigger polarity, gating signal, trigger mode ;Timing control module is divided into four modules, delay, pulse duration, pulse interval and pulse width; Output module includes the choice of pulse polarity, the output voltage or current mode, pulse amplitude, baseline, trigger light.

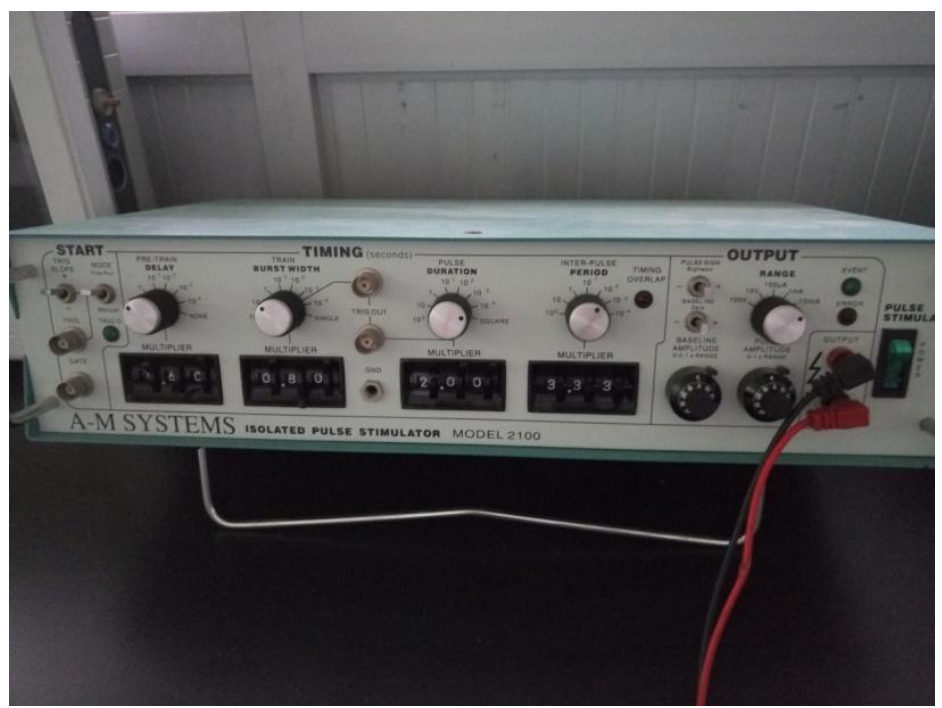

Figure 1. A-M electrical stimulator

This experiment adopts two-way symmetrical pulse wave, the frequency of superficial muscle $30 \sim 60 \mathrm{hz}$, the frequency of deep muscle $10 \sim 20 \mathrm{hz}$, pulse duration was $0.2 \sim 0.5 \mathrm{~ms}$, on-off power $/ 1 \sim 5$, pulse width is $0.8 \sim 1.8 \mathrm{~s}$, current is not more than $100 \mathrm{ma}$, respectively of flexor and extensor hands to muscle stimulation on the surface, adjust suitable current parameters and appropriate stimulation, finally realizes the hand grasping movements.

\section{The Selection of Electrical Stimulator Parameters}

Hand muscle has deep and shallow two types, electrical stimulator stimulates muscle should fully consider the two muscles of different experimental methods, so each muscle respectively set parameters of two different stimulation, otherwise it will affect the whole experiment results. The selection of test parameters have the following six points.

1. The choice of the waveform, the whole experiment that adopt double square wave, can prevent current accumulation effect. If using bipolar asymmetric wave, the cathode is equivalent to the active electrode can make muscle contractions, at this time the cathode with high concentrations of negatively charged ions, can cause to the polarization phenomenon, can cause nerve impulses or action potential, prone to the phenomenon of current burn skin. In addition, duplex wave, a wave can produce stimulation, another phase can be regarded as balance wave, can reduce electrical stimulation caused by the electrochemical action.

2. The choice of frequency: for shallow muscle, with $30 \sim 60 \mathrm{hz}$, the deep muscles, with $10 \sim 20 \mathrm{hz}$.

3. The pulse duration: $0.2 \sim 0.5 \mathrm{~ms}$.

4. The polarization time: electricity: $1: 2 \sim 5$. 
5. Group pulse width: $0.8 \sim 1.8 \mathrm{~s}$.

6. According to each person's physical fitness and endurance current amplitude factors such as different make reasonable adjustments.

\section{The Implementation of The Multiplex Way}

Because of this experiment adopts the functional electrical stimulation instrument is an instrument of single channel of functional electrical stimulation, but to stimulate the muscles usually needs to stimulate location where more than one place, so you need to set the current amplitude more than one, you need to output the single channel current amplitude in a certain way into required multiple current. As is shown in figure 2, figure 3, using PROTEL software to design the ten channels of the circuit board. According to the output of the electrical stimulator, the circuit board in the front and rear of the model are divided into two different forms: voltage mode and current mode, respectively set up ten channels, the left is the input and output pins. human body is a conductor and having certain resistance, when the output is voltage, current through the human body can also be detected .so the selection is current mode of the experiment, more simple and intuitive. Because the current is in series model, so when you need to use channels lacking ten, connect the rest of the channel with wire, otherwise it is easy to cause open circuit phenomenon.

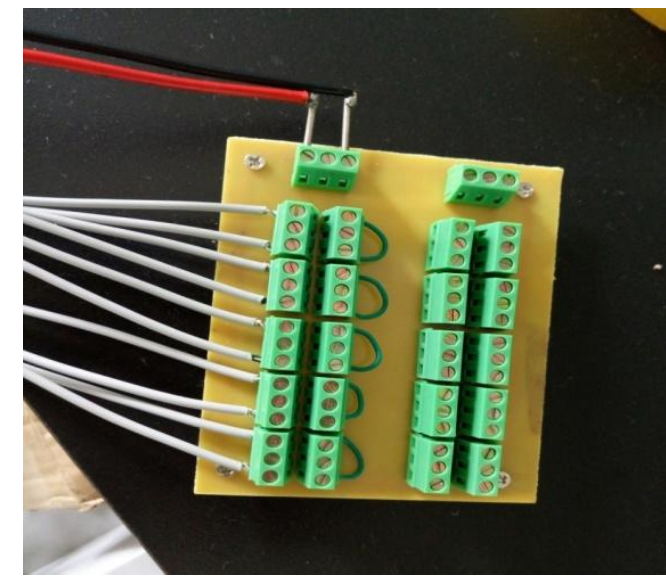

Figure 2 The face of multip lex circuit board

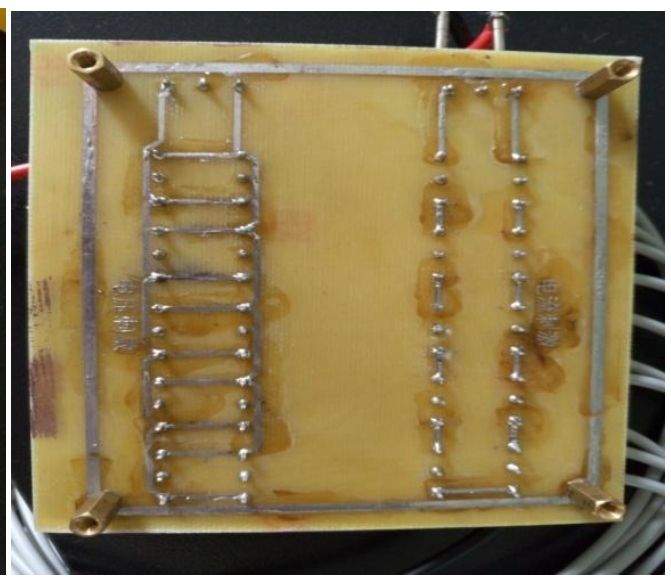

Figure 3 The opposite of mult iplex circu it board

\section{Choose the Parts of the Stimulate}

Through certain anatomical knowled ge we can know that control thumb hand muscles, mainly located in thenar muscles, divided into four parts, respectively they are thumb short abductor muscle, short for hallux flexor, thumb to thumb adductor muscle, Before the experiment, we need clean the subject's hand with $75 \%$ alcohol; purify skin surface residual oil, sweat, dander and other attached objects. Many experiments proved that the thumb to current stimulation reaction is more sensitive, so the first choose of stimulation is the single finger thumb as the subjects.

\section{The Experiment Process}

1. Select a healthy subjects who are sensitive to response to electrical stimulation.

2. For the shallow muscle, setting to the cycle $3.333 * 10^{\wedge}-2 \mathrm{~s}$, pulse duration is set to $2 * 10^{\wedge}-4 \mathrm{~s}$, group pulse width is set to $0.8 \mathrm{~s}$, delay is set to $1.6 \mathrm{~s}$.For deep muscle, cycle is set to $0.1 \mathrm{~s}$, pulse duration is set to $2 * 10^{\wedge}-4 \mathrm{~s}$, pulse width is set to 
$1.0 \mathrm{~s}$, the delay is set to $3.0 \mathrm{~s}$. That takes the double wave, the pulse output adjustment to current mode.

3. Attach the surface of electrodes respectively on thumb short abductor muscle, short for hallux flexor, on the thumb of palm thumb adductor muscle, and muscle as a positive stimulus, around these muscles in labeled as a surface electrode as cathode, forming a closed loop, watching the movement of the thumb.

Electrical stimulation to realize finger grip and three point to grasp and to stimulate the thumb grip in the same way, as shown in figure 4, figure 5, is no longer here

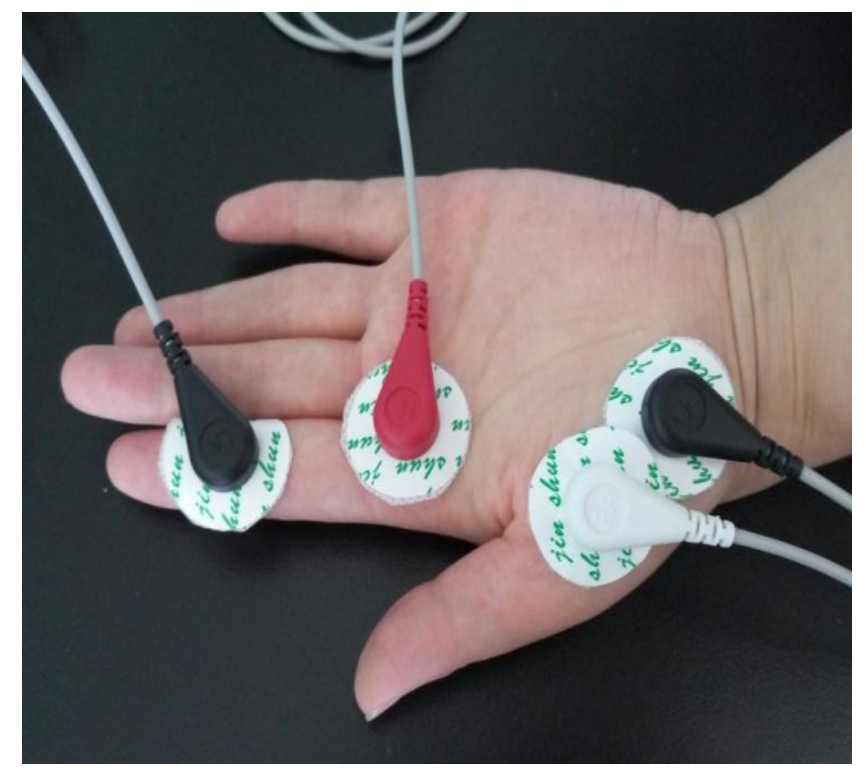

Figure 4 the surface electrodes of two re fers grasping

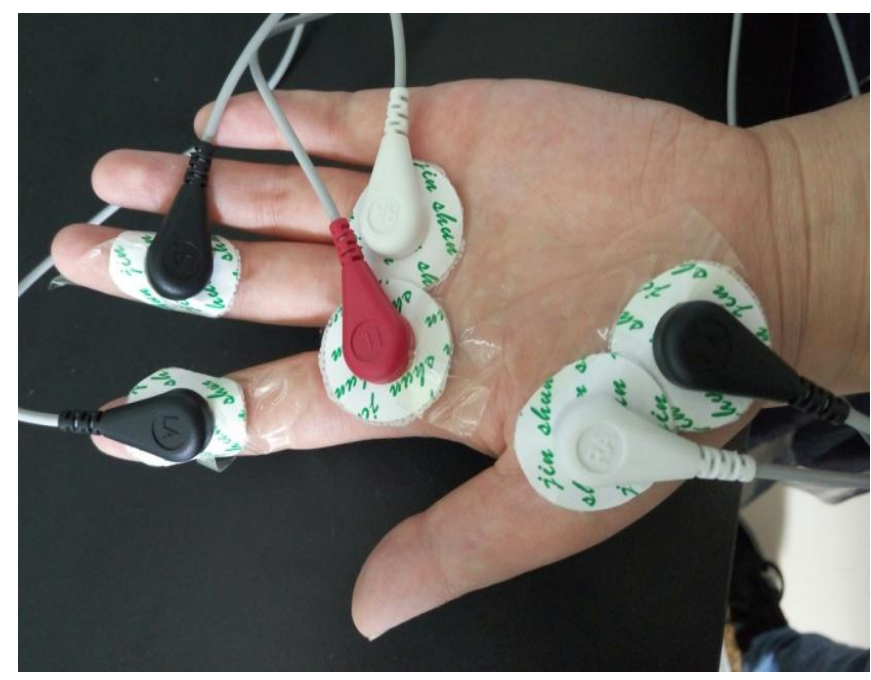

Figure 5 the surface electrodes of three refers grasping

\section{Final Analysis}

A single stimulus thumb grip conclusion is as follows. Thumb short flexor muscle of thumb short abductor muscle and shallow muscle, when the surface of electrodes on the muscles, the functional electrical stimulation device according to the shallow muscle parameter Settings, giving appropriate current stimulation, muscles 
contraction makes the thumb to outreach or inside of the bend. Thumb on palm thumb adductor muscle and belong to the deep muscles, the functional electrical stimulation device according to the deep muscles parameter settings, giving appropriate current stimulation, thumb bends to volar or lateral contraction.

And when after many times of the finger grip functional electrical stimulation experiment found that: for the hand muscle on the surface of the muscle, when more than $30 \mathrm{~mA}$ current amplitude, hand muscle on the surface of the small muscles and produce movement stimulated; For big muscles, the surface of the arm when the current rate of more than $50 \mathrm{~mA}$, the arm muscle also can produce movement due to thestimulus.

The surface of electrodes touched on the thumb short abductor muscle, under the condition of keeping other parameters constant, the delay time were set to $0.8 \mathrm{~s}, 1.6 \mathrm{~s}$, $2.4 \mathrm{~s}, 3.2 \mathrm{~s}, 4.0 \mathrm{~s}$, we can found that the thumb outreach just began a tremble, as delay extended gradually, the thumb outreach more and more stable, shaking will not happen. If the $0.8 \mathrm{~s}$ delay unchanged and the group pulse width were set to $0.8 \mathrm{~s}, 1.0 \mathrm{~s}$, $1.2 \mathrm{~s}, 1.5 \mathrm{~s}, 1.8 \mathrm{~s}$, along with the pulse width of the group of more and more wide, finger vibration amplitude is bigger and bigger. If remain the same delay and pulse width, pulse duration, respectively, set up to $10^{\wedge}-4 \mathrm{~s}, 3 * 2 * 10^{\wedge} 10^{\wedge} \wedge^{\wedge}-4 \mathrm{~s}, 4 * 4 \mathrm{~s}$, $5 * 10-4$ s.It can be seen from the change of the thumb.As the pulse duration increases gradually fingers of dithering amplitude is bigger and bigger. The group delay, pulse width and pulse duration remains the same, will cycle were set to $1.67 * 10^{\wedge} 2 \mathrm{~s}, 2.0$ $* 10^{\wedge} 2 \mathrm{~s}, 2 \mathrm{~s}, 2.5 * 10^{\wedge}-3.0 * 10^{\wedge} 2 \mathrm{~s}, 3.333 * 10^{\wedge} 2 \mathrm{~s}$. From the perspective of the movement of the thumb, the pulse cycle is more and more big, the jitter amplitude of the thumb is more and more small. So in general, the deep muscle and shallow separately set parameters are the most effective. Muscles in hand and forearm also need separate parameter settings. When the current amplitude also need separate debugging, so as to ensure the effectiveness of the stimulus, or certain influence on the accuracy of experimental results.

After many grasping electrical stimulation experiment of three fingers' results can be seen: for the hand muscle on the surface of the small muscles, when the exciting current amplitude over $35 \mathrm{ma}$, hand on the surface of the muscle small motion can be due to stimulate, for big muscle on the surface of the arm, when the current amplitude over $55 \mathrm{ma}$, arm on the surface of the large muscle also can produce movement due to the current stimulus.

\section{Conclusion}

This experiment through a frequency modulation, amplitude modulation, adjustable duty ratio of functional electrical stimulation to produce different frequency, amplitude, duty cycle of the electrical stimulation signals to stimulate the muscles and hand forearm muscle and at the same time observe corresponding motion, observing the palm found some concrete experimental data, a certain degree of general electrical stimulation signal information characteristics and finger gestures, basically realized by applying the known electrical stimulation signal to the palm to achieve a single movement, finger grasping action and three thumb gripping action of the three basic action. Especially the movement of the thumb is obvious.For later by electrical stimulation to achieve more complex hand movement laid the foundation and theoretical basis of this new subject. 


\section{Reference}

[1] Jeannerod M. The timing of natural prehension movements Journal of Motor Behavior, 1984,16:235-254.

[2] Dou HF, KK .Lee TH, et al. Iterative learning feedback control of human limbs via functional electrical stimulation [J]. Control Eng Pract.1999,(7):315-325.

[3] Ming D, Bai Y, Liu X, ct al. A gait stability investigation into FES-assisted paraplegic walking based on the walker tipping index [J].J Neural Eng, 2009, 6:066007.

[4] Meng F, Tong KY, Chan ST, et al. BCI-FES rehabilitation system based on CPG and BCI technology for locomotion: A preliminary study [C]. Lecture Notes in Computer Science, 2009, 5928:1073-1084.

[5] Zhang DG, Liu GQ, Huang G, et al. A hybrid FES rehabilitation system based on CPG and BCI technology for locomotion: A preliminary study [C]. Lecture Notes in Computer Science, 2009, 5928:1073-1084.

[6] Lan N, Crago PE, Chizeck HJ. Control of end-point forces of a multi-joint limb by functional neuromuscular stimulation [J]. IEEE Trans Biomed Eng, 1991, 38(10):953-965.

[7] He JP, Levine WS. Loeb GE. Feedback gains for correcting small perturbation of a standing posture [J].IEEE Trans Automatic Control, 1991, 36(3):322-332.

[8] Zhang DG, Zhu KY. Modeling biological motor control for human locomotion with functional electrical stimulation [J]. Biol Cybern, 2007, 96:79-97. 